\title{
Consensus Tracking of Multiagent Systems with Time-Varying Reference State and Exogenous Disturbances
}

\author{
Hong-yong Yang, Hai-lin Zou, Hui-xia Liu, Fei Liu, Mei Zhao, and Fujun Han \\ School of Information and Electrical Engineering, Ludong University, Yantai 264025, China \\ Correspondence should be addressed to Hong-yong Yang; hongyongyang@yahoo.com
}

Received 18 December 2013; Accepted 11 March 2014; Published 10 April 2014

Academic Editor: Ge Guo

Copyright ( 2014 Hong-yong Yang et al. This is an open access article distributed under the Creative Commons Attribution License, which permits unrestricted use, distribution, and reproduction in any medium, provided the original work is properly cited.

\begin{abstract}
The tracking control of multiagent dynamical systems with exogenous disturbances is studied. A path following algorithm with a time-varying reference state is proposed, and the path tracking of multiagent systems with exogenous disturbance is analyzed. Under the influence of the disturbances, a disturbance observer is developed to estimate the exogenous disturbances. Asymptotical consensus of the multiagent systems with time-varying reference state and exogenous disturbances under the disturbance observers-based control (DOBC) can be achieved for fixed and switching topologies. Finally, by applying an example of multiagent systems with switching topologies and exogenous disturbances, the consensus tracking of multiagent systems with time-varying reference state is reached under the DOBC with the designed parameters.
\end{abstract}

\section{Introduction}

Recently, the distributed cooperation problems of multiagent systems have attracted many researchers from a broad range of disciplines including physics, biology, computer science, and control engineering. This is partly because of extensive application in many areas such as collaborative control of mobile robots, unmanned air vehicles (UAVs), autonomous underwater vehicles (AUVs), and automated highway systems [1-10].

Collective behaviors of autonomous individuals have been extensively studied from different application fields. Consensus that a team of agents gradually reaches an agreement on a common reference value by negotiating with their neighbours has attracted more and more attention recently. Based on the neighbour-based swarm model proposed by Vicsek et al. [11], the theoretical frameworks for solving consensus problems for networked distributed dynamic systems were introduced in [3-5]. Further extensions of this work were presented in $[6-9,12-19]$ with directed information flow in networks. In the current studies of the agent-related problems, leader-following is one of the mainly attended topics $[6,12-14,20-25]$. In practice, multiagent systems typically need distributed sensing and control due to the constraints on, or the confluence of actuation, communication and measurement.

In the current studies, many variables of the leader in multiagent systems are researched. Distributed estimation via observers for multiagent cooperation has been an important topic in the study of agent networks. Peng and Yang studied a leader-following problem for multiagent systems with a time-varying leader and time-varying delays [20]. Hong et al. designed the distributed observers for first-order/secondorder multiagent systems where an active leader moves with an unknown sates and velocity [21,22]. Ren proposed consensus tracking algorithms under a directed information topology and with a time-varying consensus reference state [25]. In [12], Ren extended the results on tracking algorithms to the case with switching interaction topology. Low gain feedback-based distributed consensus protocols were developed in [13]; semiglobal leader-following consensus of linear multiagent systems with input saturation was investigated. In [14], adaptive leader-following consensus control was presented; the consensus of multiagent systems using model reference adaptive control approach was studied.

In this paper, we investigate the path tracking of multiagent systems with time-varying reference state and exogenous disturbances. By viewing the time-varying reference state 
as virtual leader, all agents eventually move ahead at a desired tracking under a disturbance observer-based control (DOBC). Disturbance observers (DOs) for dynamic systems have been studied and applied in engineering over two decades. With the development of DOs for robot control independently, DOs have been used in many mechanistic dynamic systems including disk drivers, machining centers, dc/ac motors, and manipulators [26-30]. Oh and Chung first improved a disturbance observer in robots using the information of nonlinear inertial coupling dynamics [26]. Wei et al. $[28,29]$ proposed a DOBC for a class of multiinputmultiple-output nonlinear system subject to disturbances. Guo et al. [27, 30] developed a DOBC for unknown constant and applied it to missile systems.

In this paper, we apply the method of DOBC to stabilize the states of multiagent systems with time-varying reference state and exogenous disturbances; this is the novelty of the paper. The rest of the paper is organized as follows. In Section 2, some preliminaries are briefly outlined. The path tracking of multiagent systems with exogenous disturbances is studied for fixed topologies and switching topologies in Section 3. Design procedure of DOBC for dynamics systems is proposed and the performances of DOBC are shown by computer simulation in Section 4. Finally, conclusions are drawn in Section 5.

\section{Preliminaries}

A weighted connected graph $\mathscr{G}=\{V, E, A\}$ of order $n$ consists of a set of vertices $V=\left\{v_{1}, v_{2}, \ldots, v_{n}\right\}$, a set of edges $E \subseteq V \times V$, and an adjacency matrix $A=\left[a_{i j}\right] \in R^{n \times n}$ with weighted adjacency elements $a_{i j} \geq 0$. The node indexes belong to a finite index set $I=\{1,2, \ldots, n\}$. An edge of the weighted diagraph $\mathscr{G}$ is denoted by $e_{i j}=\left(v_{i}, v_{j}\right) \in E$ indicating that node $i$ can influence node $j$ and not vice versa. Assume that the adjacency element $a_{i j}>0$ for $e_{i j} \in E$; otherwise, $a_{i j}=$ 0 . If $a_{i j}=a_{j i}$ for all $i, j \in I$, the graph $\mathscr{G}$ is an undirected graph.

The set of neighbors of node $i$ is denoted by $N_{i}=\{j \in$ $\left.I: a_{i j}>0\right\}$. Let $\mathscr{G}$ be a weighted graph without self-loops; that is, $a_{i i}=0$, and let matrix $D$ be a diagonal matrix with the elements $d_{i}$ along the diagonal, where $d_{i}=\sum_{j=1}^{n} a_{i j}$ is the outdegree of the node $i$. The Laplacian matrix of the weighted graph is defined as $L=D-A$.

A directed path is a sequence of edges in a directed graph $\left(i_{1}, i_{2}\right),\left(i_{2}, i_{3}\right), \ldots,\left(i_{i-1}, i_{l}\right)$. There exists a directed path from node $i$ to node $j$; then node $i$ is said to be reachable node to node $j$. For any node $i$, if there is a path to every node in digraph $\mathscr{G}$, then node $i$ is called a globally reachable node in $\mathscr{G}$.

A dynamical equation of multiagent systems was considered in [12],

$$
\dot{x}_{i}(t)=v_{i}(t), \quad i=1, \ldots, n
$$

where $x_{i} \in R^{m_{1}}$ and $v_{i} \in R^{m_{1}}$ are the position and velocity control input, respectively, of agent $i$. In order to calculate easily, we suppose $m_{1}=1$. A consensus algorithm for singleintegrator dynamics was proposed as

$$
v_{i}(t)=-\sum_{j=1}^{n} a_{i j}\left(x_{i}(t)-x_{j}(t)\right),
$$

where $a_{i j}$ is the $(i, j)$ entry of the adjacency matrix. With algorithm (2), consensus is achieved by the team of agents if, for all initial state $x_{i}(0),\left\|x_{i}(t)-x_{j}(t)\right\| \rightarrow 0$ as $t \rightarrow \infty$, for all $i, j=1, \ldots, n$.

Lemma 1 (see [12]). Multiagent systems composed of $n$ agents with algorithm (1)-(2) can achieve consensus asymptotically if and only if directed graph of agents has a globally reachable node. Specifically, $x_{i}(t) \rightarrow \sum_{j=1}^{n} w_{j} x_{j}(0)$ as $t \rightarrow \infty$, where $w=\left[w_{1}, \ldots, w_{n}\right]^{T} \geq 0,1_{n}^{T} w=1$, and $L^{T} w=1$.

\section{Consensus Algorithm of Multiagent Systems with Time-Varying Reference State}

3.1. Dynamical Networks with Disturbances and Time-Varying Reference State. The moving state of system (1)-(2) is affected by the initialized values of multiagent systems. If the initialized values are set up randomly, the asymptotic state cannot be converged to an expected path based on Lemma 1. Moreover, unmodeled dynamics and parametric variations as well as external disturbances widely exist in practical processes, which will influence the moving track of the systems. In this paper, we suppose the system (1)-(2) with exogenous disturbances and develop a composite controller to bring the moving state of multiple agents to an expected path tracking. The multiagent systems with exogenous disturbance are described by, for all $i \in I$,

$$
\dot{x}_{i}(t)=u_{i}+d_{i}
$$

where $u_{i} \in R$ and $d_{i} \in R$ are the control input and external disturbance, respectively. It is supposed that the disturbance $d_{i}$, for $i=1, \ldots, n$, is generated by a linear exogenous system

$$
\begin{aligned}
& \dot{\xi}_{i}(t)=W \xi_{i}(t), \\
& d_{i}(t)=C \xi_{i}(t),
\end{aligned}
$$

where $\xi_{i} \in R^{m}$ is the internal state of the exogenous system, $W \in R^{m \times m}$ and $C \in R^{1 \times m}$ are the matrices of the disturbance system, and $(W, C)$ is observable.

Suppose that we want to stabilize multiagent systems (3) onto an expected reference consensus state defined by

$$
\dot{x}_{0}(t)=v_{0}(t), \quad \dot{v}_{0}(t)=u_{0}(t),
$$

where $u_{0}(t)=f\left(t, v_{0}\right)$ is the velocity function of the reference and $f(\cdot, \cdot)$ is bounded, piecewise continuous in $t$, and locally Lipschitz in $v_{0}$.

In this paper, we discuss the path following of the multiagent dynamical systems with disturbances. The objective is to design a DOBC technique such that the expected reference path (5) on the composite controller is achieved asymptotically. 
3.2. Consensus Tracking Control for Multiagent Systems without Disturbances. In this subsection, we suppose there is no disturbance or the disturbance is measurable. The tracking consensus of system (3) is studied by designing a controller. In order to realize the expected path consensus of systems (3), some controllers may be designed and applied to the systems.

Here, the controlled system (3) without disturbance can be described by, for all $i=1, \ldots, n$,

$$
\dot{x}_{i}(t)=u_{i} .
$$

The consensus tracking control is given as

$$
\begin{array}{r}
u_{i}(t)=-\sum_{j=1}^{n} a_{i j}\left(x_{i}(t)-x_{j}(t)\right) \\
-b_{i}\left(x_{i}(t)-x_{0}(t)\right)+v_{i}(t), \\
\dot{v}_{i}(t)=u_{0}(t)+\gamma\left(\sum_{j=1}^{n} a_{i j}\left(x_{j}(t)-x_{i}(t)\right)\right. \\
\left.-b_{i}\left(x_{i}(t)-x_{0}(t)\right)\right),
\end{array}
$$

where the control gain $b_{i} \geq 0$, for all $i=1, \ldots, n$, and at least there is $i_{0} \in I$ with $b_{i_{0}}>0 . \gamma>0$ is the control gain to be designed. Let $B=\operatorname{diag}\left\{b_{1}, \ldots, b_{l}, b_{l+1}, \ldots, b_{n}\right\}$. Based on the method in [21], a distributed observer $\dot{v}_{i}(t)$ is developed to compensate the consensus tracking controller in (7).

Lemma 2 (see [12]). Assume the directed graph of $n$ agents and one leader with leader as a globally reachable node. Then, the matrix $L+B$ is positively stable, where $L$ is the Laplacian matrix of the connected graph of $n$ agents.

Lemma 3 (see [31], (Hermite-Biehler Theorem)). Suppose the polynomial

$$
p(s)=p_{0}+p_{1} s+\cdots+p_{n} s^{n}
$$

Substituting $s=\mathbf{j} \omega$ (where $\mathbf{j}$ is imaginary unit) into the polynomial $p(s)$ yields

$$
p(\omega)=m(\omega)+\mathbf{j} n(\omega) .
$$

Then, the polynomial $p(s)$ is Hurwitz stable if and only if the related pair $m(\omega), n(\omega)$ is interlaced, and $m(0) n^{\prime}(0)-$ $m^{\prime}(0) n(0)>0$.

Theorem 4. Assume that multiagent systems are composed of $n$ agents with a globally reachable node, and some globally reachable nodes can receive the information from the reference agent. Then, based on the consensus tracking control (7), the multiagent systems (6) without disturbance can asymptotically reach the consensus reference state, if the control gain satisfies

$$
\gamma<\min \left\{\frac{\operatorname{Re}\left(\lambda_{i}\right)}{\left(\operatorname{Im}\left(\lambda_{i}\right)\right)^{2}}\left|\lambda_{i}\right|^{2}, i \in I\right\},
$$

where $\lambda_{i}$ is the characteristic value of the matrix $L+B$.
Proof. Let $\delta_{i}(t)=x_{i}(t)-x_{0}(t), \widetilde{v}_{i}(t)=v_{i}(t)-v_{0}(t)$. Then, the multiagent systems (6) with the control (7) can be rewritten as, for all $i=1, \ldots, n$,

$$
\begin{aligned}
& \dot{\delta}_{i}(t)=-\sum_{j=1}^{n} a_{i j}\left(\delta_{i}(t)-\delta_{j}(t)\right)-b_{i} \delta_{i}(t)+\widetilde{v}_{i}(t), \\
& \dot{\widetilde{v}}_{i}(t)=\gamma\left(\sum_{j \in N_{i}} a_{i j}\left(\delta_{j}(t)-\delta_{i}(t)\right)-b_{i} \delta_{i}(t)\right) .
\end{aligned}
$$

Let $\delta=\left[\delta_{1}, \ldots, \delta_{n}\right]^{T}, \widetilde{v}(t)=\left[\widetilde{v}_{1}, \ldots, \widetilde{v}_{n}\right]^{T}$; we have

$$
\begin{aligned}
& \dot{\delta}(t)=-\bar{L} \delta(t)+I_{n} \widetilde{v}(t), \\
& \dot{\tilde{v}}(t)=-\gamma \bar{L} \delta(t),
\end{aligned}
$$

where $\bar{L}=(L+B)$ and $I_{n}=\operatorname{diag}\{1, \ldots, 1\}$. Taking the Laplace transforms of the systems (12) yields

$$
\begin{aligned}
& s \Delta(s)-\delta(0)=-\bar{L} \Delta(s)+I_{n} V(s), \\
& s V(s)-v(0)=-\gamma \bar{L} \Delta(s),
\end{aligned}
$$

where $\Delta(s), V(s)$ is the Laplace transforms of $\delta(t), \widetilde{v}(t)$, respectively. Then, we can obtain the characteristic equation of system (12):

$$
\operatorname{det}\left(s I_{2 n}-\left(\begin{array}{cc}
-\bar{L} & I_{n} \\
-\gamma \bar{L} & 0
\end{array}\right)\right)=0
$$

Equation (14) is equivalent to

$$
\operatorname{det}\left(s^{2} I_{n}+(s+\gamma) \bar{L}\right)=0 .
$$

The characteristic equation can be rewritten as

$$
\prod_{i=1}^{n}\left(s^{2}+(s+\gamma) \lambda_{i}\right)=0,
$$

where $\lambda_{i}(i=1,2, \ldots, n)$ is the characteristic value of $\bar{L}$. Let $\lambda_{i}=a+\mathrm{j} b$, where $a>0$ from Lemma 2. Suppose $p(s)=$ $s^{2}+(s+\gamma)(a+\mathbf{j} b)$; let $s=\mathbf{j} \omega$; we have

$$
p(\omega)=m(\omega)+\mathbf{j} n(\omega),
$$

where $m(\omega)=-\omega^{2}-b \omega+\gamma a, n(\omega)=a \omega+\gamma b$. It can be obtained easily, $m(0) n^{\prime}(0)-m^{\prime}(0) n(0)>0$. Since the polynomial $m(\omega)$ has two distinct roots,

$$
m_{1,2}=\frac{-b \pm \sqrt{b^{2}+4 \gamma a}}{2} .
$$

The polynomial $n(\omega)$ has one $\operatorname{root} n=-\gamma b / a$. From Lemma 3, the polynomial $p(s)$ is Hurwitz stable if and only if the related pair $m(\omega), n(\omega)$ is interlaced, and $m(0) n^{\prime}(0)-m^{\prime}(0) n(0)>0$. Then, we present the following inequality:

$$
\frac{-b-\Xi}{2}<-\frac{\gamma b}{a}<\frac{-b+\Xi}{2}
$$


where $\Xi=\sqrt{b^{2}+4 \gamma a}$. Suppose the Laplacian matrix $L$ is symmetric; that is, $b=0$; at this time, the above inequality holds. For nonsymmetric Laplacian matrix $b \neq 0$, we get $\gamma b^{2}<$ $a\left|\lambda_{i}\right|^{2}$; then

$$
\gamma<\frac{a}{b^{2}}\left|\lambda_{i}\right|^{2}
$$

Based on Lemma 3, the polynomial $p(s)$ is Hurwitz stable if the inequality (20) holds. Therefore, the system (6) is asymptotically stable; that is, $\lim _{t \rightarrow+\infty} \delta(t)=0$. The multiagent systems (6) without disturbance can asymptotically reach the consensus reference state. The proof of Theorem 4 is finished.

Remark 5. Suppose the topology of multiagent systems is symmetrical; the multiagent systems (6) without disturbance can asymptotically reach the consensus reference state for any control gain $\gamma>0$.

Remark 6. Since the characteristic value $\lambda_{i}=a+\mathbf{j} b$, we have $b^{2}<\left|\lambda_{i}\right|^{2}$. If $\gamma<a$, the consensus tracking of multiagent systems can be achieved.

3.3. DOBC for Multiagent Systems with Fixed Topologies. It is supposed that the states of the system (3) can be obtained and the disturbance is generated by a linear exogenous system (4). A disturbance observer is proposed to estimate the unknown disturbance $d_{i}$ in system (3), for all $i \in I$,

$$
\begin{gathered}
\dot{z}_{i}(t)=(W+K C)\left(z_{i}(t)-K x_{i}(t)\right)+K\left(L_{i} x(t)+u_{i}(t)\right), \\
\widehat{\xi}_{i}(t)=z_{i}(t)-K x_{i}(t), \\
\widehat{d}_{i}(t)=C \widehat{\xi}_{i}(t),
\end{gathered}
$$

where $z_{i} \in R^{m}$ is the internal state variable of the observer and $\widehat{\xi}_{i}$ and $\widehat{d}_{i}$ are the estimates of $\xi_{i}$ and $d_{i}$, respectively. $K \in R^{m \times 1}$ is the observer gain matrix to be designed. $L_{i} \in R^{1 \times n}$ can be obtained from (3) satisfying

$$
\dot{x}_{i}(t)=L_{i} x(t)+u_{i}(t)+d_{i}(t),
$$

where $L_{i}$ is a submatrix of $L$ obtained by selecting the $i$ th row in $L$, and $x(t)=\left[x_{1}(t), \ldots, x_{n}(t)\right]^{T}$. Let

$$
e_{i}(t)=\xi_{i}(t)-\widehat{\xi}_{i}(t), \quad i=1, \ldots, n,
$$

be the estimation error; based on (3), (4), and (21), it is shown that

$$
\dot{e}_{i}(t)=(W+K C) e_{i}(t) .
$$

Then, we can get that the disturbance observers (21) can exponentially track the disturbance from the following Lemma.

Lemma 7. Consider system (3) under the disturbance generated by exogenous system (4). The error system (24) is globally exponentially stable, if there exists gain matrix $K$ satisfying

$$
W+K C<0
$$

where $W+K C$ is negative definite.
Proof. Since $(W, C)$ is observable in (4), there exists gain matrix $K$ satisfying $W+K C<0$.

Theorem 8. Consider multiagent systems (3) with globally reachable nodes and the exogenous disturbances (4). Suppose that some globally reachable nodes can receive the information from the reference tracking; then the multiagent dynamical systems (3) under the tracking controls (7) and the disturbance observers (21) can reach expected path tracking asymptotically, if the control gain of tracking controls (7) satisfies the inequations (10) and the gain matrix $K$ of the disturbance observers (21) satisfies the inequations (25).

Proof. Because of the disturbances in the system, the control $u(x, d)$ will depend on the disturbance $d$. Here, we will apply $\widehat{d}$ instead of $d$; the composite controller is designed as

$$
\begin{aligned}
& u_{i}(t)=-\sum_{j=1}^{n} a_{i j}\left(x_{i}(t)-x_{j}(t)\right)-b_{i}\left(x_{i}(t)-x_{0}(t)\right) \\
&+v_{i}(t)-\widehat{d}_{i}(t), \\
& \dot{v}_{i}(t)= u_{0}(t)+\gamma\left(\sum_{j \in N_{i}} a_{i j}\left(x_{j}(t)-x_{i}(t)\right)\right. \\
&\left.-b_{i}\left(x_{i}(t)-x_{0}(t)\right)\right) .
\end{aligned}
$$

Substituting (26) into system (3) and letting $\delta_{i}(t)=x_{i}(t)-$ $x_{0}(t)$ and $\widetilde{v}_{i}(t)=v_{i}(t)-v_{0}(t)$, respectively, we have

$$
\begin{aligned}
& \dot{\delta}_{i}(t)=\sum_{j=1}^{n} a_{i j}\left(\delta_{j}(t)-\delta_{i}(t)\right)-b_{i} \delta_{i}(t)+\widetilde{v}_{i}(t)+C e_{i}(t), \\
& \dot{\widetilde{v}}_{i}(t)=\gamma\left(\sum_{j \in N_{i}} a_{i j}\left(\delta_{j}(t)-\delta_{i}(t)\right)-b_{i} \delta_{i}(t)\right) .
\end{aligned}
$$

Let $\delta=\left[\delta_{1}, \ldots, \delta_{n}\right]^{T}, \widetilde{v}(t)=\left[\widetilde{v}_{1}, \ldots, \widetilde{v}_{n}\right]^{T}, e(t)=\left[e_{1}(t), \ldots\right.$, $\left.e_{n}(t)\right]^{T}$; the closed-loop system including the multiagent system (27) and the error dynamics (24) can be obtained as follows:

$$
\begin{gathered}
\dot{\delta}(t)=-\bar{L} \delta(t)+\Psi e(t)+I_{n} \widetilde{v}(t), \\
\dot{\tilde{v}}(t)=-\gamma \bar{L} \delta(t), \\
\dot{e}(t)=\Omega e(t),
\end{gathered}
$$

where $\bar{L}=L+B, \Psi=I_{n} \otimes C, \Omega=I_{n} \otimes(W+K C), \otimes$ is the Kronecker product. Applying the Laplace transforms of the systems (28), respectively, we yield

$$
\begin{gathered}
s \Delta(s)-\delta(0)=-\bar{L} \Delta(s)+\Psi E(s)+I_{n} V(s), \\
s V(s)-v_{0}=-\gamma \bar{L} \Delta(s), \\
s E(s)-e(0)=\Omega E(s),
\end{gathered}
$$




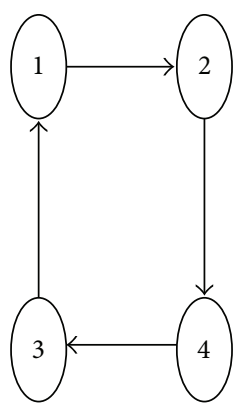

(a)

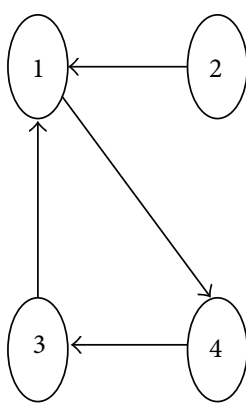

(b)

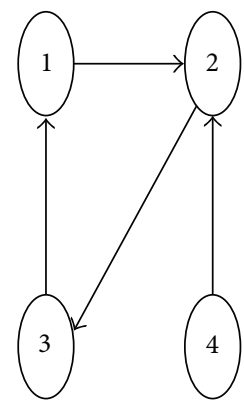

(c)

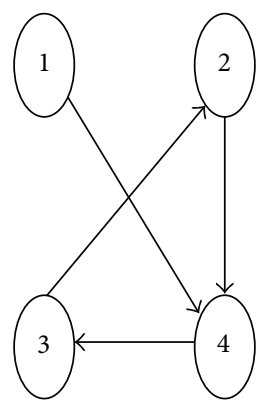

(d)

FIGURE 1: Switching graph of multiagent systems.

where $\Delta(s), V(s), E(s)$ is the Laplace transforms of $\delta(t)$, $\widetilde{v}(t), e(t)$, respectively. Then, we can obtain the characteristic equation of the systems (28):

$$
\operatorname{det}\left(s I_{2 n+m n}-\Theta\right)=0,
$$

where

$$
\Theta=\left(\begin{array}{ccc}
-\bar{L} & I_{n} & \Psi \\
-\gamma \bar{L} & 0 & 0 \\
0 & 0 & \Omega
\end{array}\right) .
$$

Equation (30) is equivalent to

$$
\begin{gathered}
\operatorname{det}\left(s I_{2 n}-\left(\begin{array}{cc}
-\bar{L} & I_{n} \\
-\gamma \bar{L} & 0
\end{array}\right)\right)=0, \\
\operatorname{det}\left(s I_{m n}-\Omega\right)=0 .
\end{gathered}
$$

Suppose the control gain of tracking controls (7) satisfies the inequations (10) and the gain matrix $K$ of the disturbance observers (21) satisfies the inequations (25); the characteristic values of (32) and (33) are negative from Theorem 4 and Lemma 7. Therefore, the system (3) is asymptotically stable with DOBC. Then, we can obtain that the consensus of the multiagent systems (3) can be achieved.

3.4. DOBC for Multiagent Systems with Switching Topologies. Consider system (3) with switching topologies $\left\{\mathscr{G}_{s}: s=\right.$ $\left.\sigma(t) \in \Pi_{0}\right\}$, where $\Pi_{0} \subset Z$ is a finite index set and $\sigma(t)$ is a switching signal determining the network topology with interconnection. Under arbitrary switching signal, the switching system induced by (3) takes the following form:

$$
\dot{x}_{i}(t)=\sum_{j \in N_{i}(t)} a_{s, i j}\left(x_{j}(t)-x_{i}(t)\right)+u_{i}+d_{i},
$$

where $s=\sigma(t) \in \Pi_{0}$ and $a_{s, i j} \geq 0$ denotes the weighted value between node $i$ and node $j$ at switching signal $s$. Let $L_{s}$ be the Laplacian matrix of the switching topology $\mathscr{G}_{s}$.

Theorem 9. Consider multiagent systems (34) with switching topologies $\mathscr{G}_{s}$ and the disturbance (4). At every time interval, the switching topologies $\mathscr{G}_{s}$ have globally reachable nodes. Suppose that the globally reachable nodes can communicate with the reference agent; then, the multiagent dynamical systems (34) under the tracking controls (7) and the disturbance observers (21) can reach consensus reference tracking asymptotically, if the control gain of tracking controls (7) satisfies the inequations (10) and the gain matrix $K$ of the disturbance observers (21) satisfies the inequations (25).

Proof. The proof is similar to that of Theorem 8; we can obtain from (28)

$$
\dot{\Lambda}(t)=\Theta_{\sigma(t)} \Lambda(t),
$$

where

$$
\Theta_{\sigma(t)}=\left(\begin{array}{ccc}
-\bar{L}_{\sigma(t)} & I_{n} & \Psi \\
-\gamma \bar{L}_{\sigma(t)} & 0 & 0 \\
0 & 0 & \Omega
\end{array}\right),
$$

and $\Lambda=\left[\delta_{\sigma(t)}(t) \widetilde{v}_{\sigma(t)}(t) e_{\sigma(t)}(t)\right]^{T}, \Psi=I_{n} \otimes C, \Omega=I_{n} \otimes$ $(W+K C)$.

If the gain matrix $K$ of the disturbance observers (21) satisfies the inequations (25), the matrix $\Omega$ is negative definite. We can obtain that the error dynamics $\dot{e}_{\sigma(t)}=\Omega e_{\sigma(t)}$ is asymptotically stable. Similarly, the multiagent dynamical systems (34) without the disturbances under the tracking controls (7) and the disturbance observers (21) can reach consensus reference tracking. Based on the method of DOBC, the results are proved.

\section{Example Study}

Consider directed connected networks with 4 agents (Figure 1). The weighted values of the graph are given as 1 and Laplacian matrix $L_{s}$ can be obtained depending on the adjacency matrix of the switching topologies.

Case 1. Suppose that disturbances in multiagent systems do not exist (or there exist measurable disturbances). Let the initial states of agents be generated randomly in $[0,5]$, and let the expected reference track be $\dot{\bar{x}}(t)=\cos (t)+2 \cos (2 t)$ with the initialized value at random. We apply the control (7) with the control gain $b_{i}=1$ randomly, where $i=3$ for the graph (a), $i=2$ for the graph (b), $i=4$ for the graph (c), and $i=1$ for the graph (d). We can obtain that the control gain 


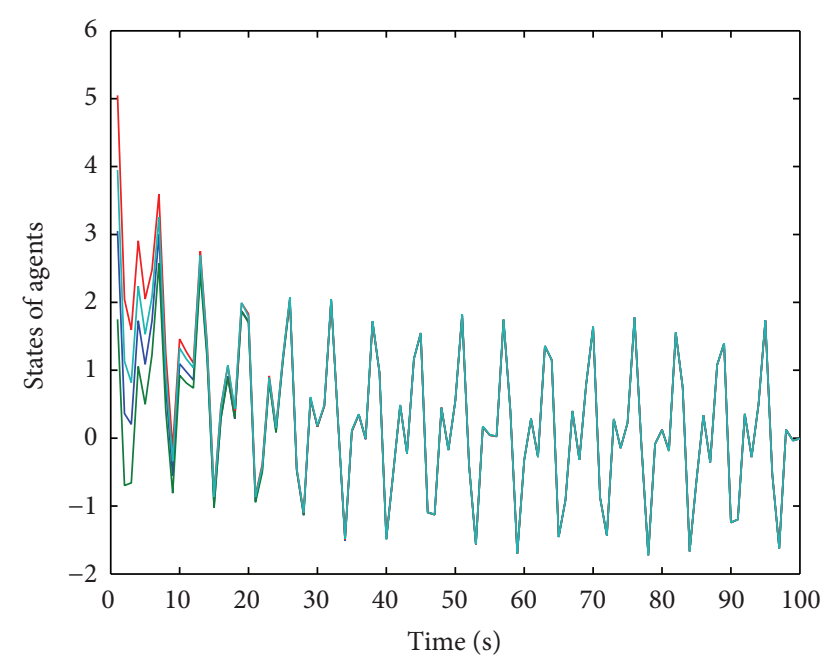

FIGURE 2: States tracking of multiagent systems without disturbance.

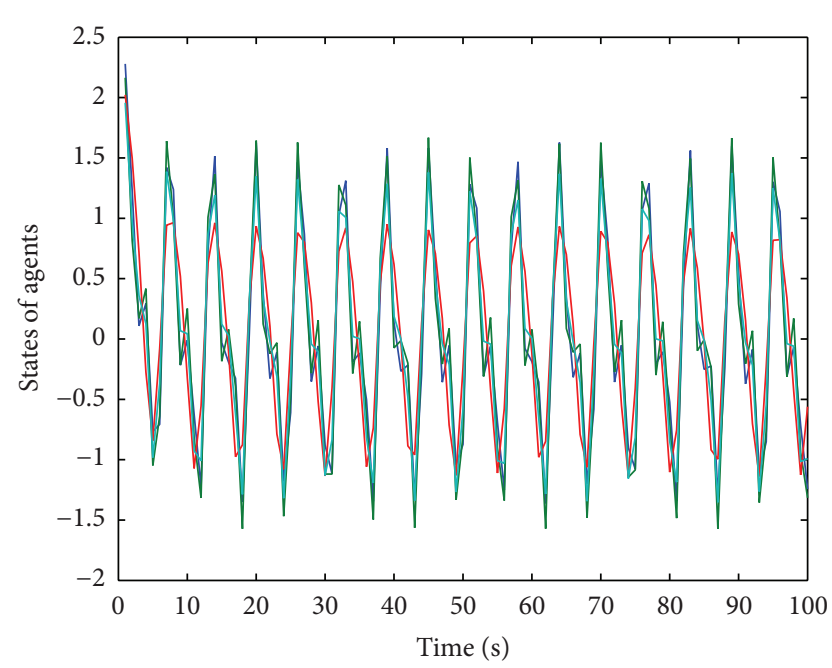

FIgURE 3: States of multiagent systems with disturbance.

$\gamma<2.22$ from Theorem 4. The moving states of multiagent systems with the control are plotted in Figure 2. We can see that the reference track is reached asymptotically.

Case 2. Consider the multiagent systems with disturbances. Suppose that the parameters of the exogenous disturbance system (4) are $W=\left[\begin{array}{cc}0 & 2 \\ -2 & 0\end{array}\right]$ and $C=[1,0]$ with initialized value $\xi_{i}=[0.5 \sin 1,0.5 \cos 1]^{T}$. In Figure 3 , the outputs of the multiagent systems with exogenous disturbances are shown.

By applying linear matrix inequalities (LMIs) packages of Matlab solving inequality (25), we can get $K=[-3,1]^{T}$. The estimation obtained by observer (16) is shown in Figure 4, where the dot line is the estimation of the exogenous disturbance. We can see that the observer exhibits excellent tracking performance. In Figure 5, the moving path of multiagent systems with DOBC is plotted, showing that the reference tracking has been achieved for multiagent systems with the exogenous disturbances under DOBC.

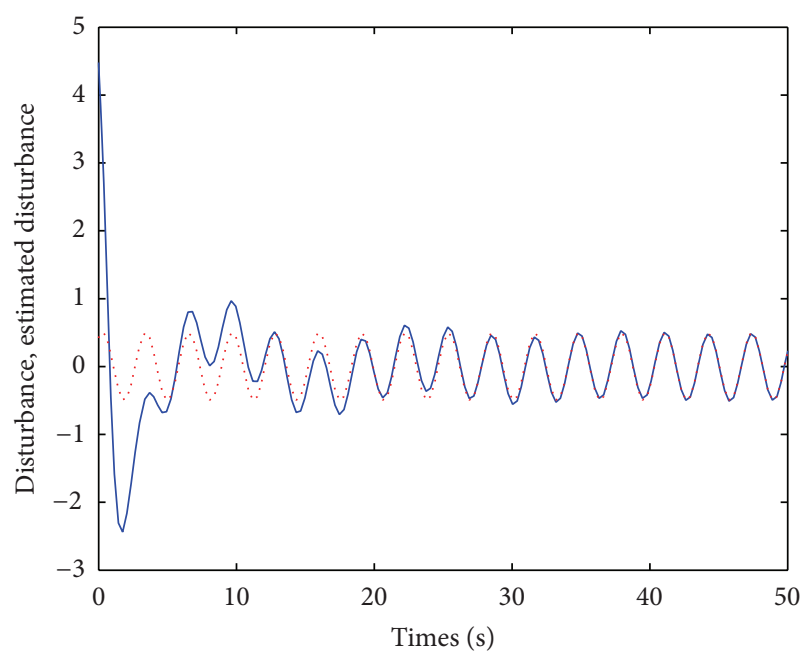

FIgURE 4: Disturbances estimated by DOBC.

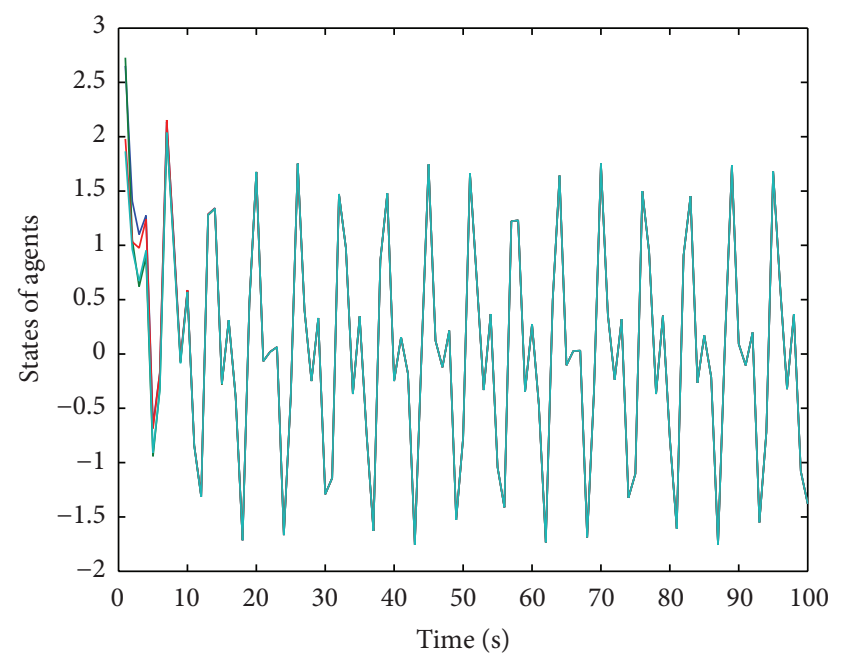

FIgURE 5: States of multiagent systems with DOBC.

\section{Conclusions}

The problem of cooperation in a team of unmanned systems with the expected path tracking is considered under the influences of disturbances. A control strategy is designed for the multiagent systems without disturbances, where multiple agents' states can be controlled to a reference path. Under the effects of the disturbances, disturbance observers are developed by an exogenous system to estimate the disturbances. The disturbance observers are integrated with the controller by replacing the disturbance in the control law with its estimation obtained from the disturbance observer. Furthermore, asymptotical consensus of the multiagent systems with disturbances under the composite controller is achieved.

\section{Conflict of Interests}

The authors declare there is no conflict of interests regarding the publication of this paper. 


\section{Acknowledgments}

This research is supported by the National Basic Research Science Program of China (under Grant 2012CB720000, 973 Program), the National Natural Science Foundation of China (under Grants 61273152, 61170161, 61174085, and 61304052), the Natural Science Foundation of Shandong Province of China (under Grant ZR2011FM017), and the Outstanding Young Research Award Fund of Shang Province of China (under Grant BS2013DX005).

\section{References}

[1] R. Olfati-Saber, J. A. Fax, and R. M. Murray, "Consensus and cooperation in networked multi-agent systems," Proceedings of the IEEE, vol. 95, no. 1, pp. 215-233, 2007.

[2] W. Ren, R. W. Beard, and E. M. Atkins, "Information consensus in multivehicle cooperative control," IEEE Control Systems Magazine, vol. 27, no. 2, pp. 71-82, 2007.

[3] J. A. Fax and R. M. Murray, "Information flow and cooperative control of vehicle formations," IEEE Transactions on Automatic Control, vol. 49, no. 9, pp. 1465-1476, 2004.

[4] A. Jadbabaie, J. Lin, and A. S. Morse, "Coordination of groups of mobile autonomous agents using nearest neighbor rules," IEEE Transactions on Automatic Control, vol. 48, no. 6, pp. 988-1001, 2003.

[5] R. Olfati-Saber and R. M. Murray, "Consensus problems in networks of agents with switching topology and time-delays," IEEE Transactions on Automatic Control, vol. 49, no. 9, pp. 15201533, 2004.

[6] H. Su, X. Wang, and W. Yang, "Flocking in multi-agent systems with multiple virtual leaders," Asian Journal of Control, vol. 10, no. 2, pp. 238-245, 2008.

[7] P. Lin, Y. Jia, J. Du, and S. Yuan, "Distributed control of multiagent systems with second-order agent dynamics and delaydependent communications," Asian Journal of Control, vol. 10, no. 2, pp. 254-259, 2008.

[8] S. Li, H. Du, and X. Lin, "Finite-time consensus algorithm for multi-agent systems with double-integrator dynamics," Automatica, vol. 47, no. 8, pp. 1706-1712, 2011.

[9] H. Du, S. Li, and C. Qian, "Finite-time attitude tracking control of spacecraft with application to attitude synchronization," IEEE Transactions on Automatic Control, vol. 56, no. 11, pp. 2711-2717, 2011.

[10] S. Li and X. Wang, "Finite-time consensus and collision avoidance control algorithms for multiple AUVs," Automatica, vol. 49, no. 11, pp. 3359-3367, 2013.

[11] T. Vicsek, A. Czirk, E. B. Jacob, I. Cohen, and O. Shochet, "Novel type of phase transition in a system of self-driven particles," Physical Review Letters, vol. 75, no. 6, pp. 1226-1229, 1995.

[12] W. Ren, "Consensus tracking under directed interaction topologies: algorithms and experiments," in Proceedings of the American Control Conference (ACC '08), pp. 742-747, Seattle, Wash, USA, June 2008.

[13] H. Su, M. Z. Q. Chen, J. Lam, and Z. Lin, "Semi-global leaderfollowing consensus of linear multi-agent systems with input saturation via low gain feedback," IEEE Transactions on Circuits and Systems-I. Regular Papers, vol. 60, no. 7, pp. 1881-1889, 2013.

[14] Y. Liu and Y. Jia, "Adaptive leader-following consensus control of multi-agent systems using model reference adaptive control approach," IET Control Theory \& Applications, vol. 6, no. 13, pp. 2002-2008, 2012.

[15] H.-Y. Yang, L. Guo, B. Xu, and J.-Z. Gu, "Collaboration control of fractional-order multiagent systems with sampling delay," Mathematical Problems in Engineering, vol. 2013, Article ID 854960, 7 pages, 2013.

[16] Y.-P. Tian and Y. Zhang, "High-order consensus of heterogeneous multi-agent systems with unknown communication delays," Automatica, vol. 48, no. 6, pp. 1205-1212, 2012.

[17] C.-L. Liu and F. Liu, "Stationary consensus of heterogeneous multi-agent systems with bounded communication delays," Automatica, vol. 47, no. 9, pp. 2130-2133, 2011.

[18] L. Ding, Q.-L. Han, and G. Guo, "Network-based leader-following consensus for distributed multi-agent systems," Automatica, vol. 49, no. 7, pp. 2281-2286, 2013.

[19] G. Guo, Y. Zhao, and G. Yang, "Cooperation of multiple mobile sensors with minimum energy cost for mobility and communication," Information Sciences, vol. 254, no. 1, pp. 69-82, 2014.

[20] K. Peng and Y. Yang, "Leader-following consensus problem with a varying-velocity leader and time-varying delays," Physica A, vol. 388, no. 2-3, pp. 193-208, 2009.

[21] Y. Hong, J. Hu, and L. Gao, "Tracking control for multiagent consensus with an active leader and variable topology," Automatica, vol. 42, no. 7, pp. 1177-1182, 2006.

[22] Y. Hong, G. Chen, and L. Bushnell, "Distributed observers design for leader-following control of multi-agent networks," Automatica, vol. 44, no. 3, pp. 846-850, 2008.

[23] R. Olfati-Saber, "Flocking for multi-agent dynamic systems: algorithms and theory," IEEE Transactions on Automatic Control, vol. 51, no. 3, pp. 401-420, 2006.

[24] H. Shi, L. Wang, and T. Chu, "Virtual leader approach to coordinated control of multiple mobile agents with asymmetric interactions," Physica D, vol. 213, no. 1, pp. 51-65, 2006.

[25] W. Ren, "Multi-vehicle consensus with a time-varying reference state," Systems \& Control Letters, vol. 56, no. 7-8, pp. 474-483, 2007.

[26] Y. Oh and W. K. Chung, "Disturbance-observer-based motion control of redundant manipulators using inertially decoupled dynamics," IEEE/ASME Transactions on Mechatronics, vol. 4, no. 2, pp. 133-146, 1999.

[27] L. Guo, C. Feng, and W. Chen, "A survey of disturbance observer-based control for dynamic nonlinear system," Dynamics of Continuous Discrete and Impulsive Systems B: Applications \& Algorithms, vol. 13, pp. 79-84, 2006.

[28] X. Wei and L. Guo, "Composite disturbance-observer-based control and terminal sliding mode control for non-linear systems with disturbances," International Journal of Control, vol. 82, no. 6, pp. 1082-1098, 2009.

[29] X. Wei, L. Wu, and N. Chen, "Composite disturbance-observerbased control and $H_{\infty}$ control for nonlinear time-delay systems," Asian Journal of Control, vol. 11, no. 4, pp. 440-443, 2009.

[30] L. Guo and W.-H. Chen, "Disturbance attenuation and rejection for systems with nonlinearity via DOBC approach," International Journal of Robust and Nonlinear Control, vol. 15, no. 3, pp. 109-125, 2005.

[31] K. Ogata, Discrete-Time Control Systems, Prentice-Hall, Englewood Cliffs, NJ, USA, 1995. 


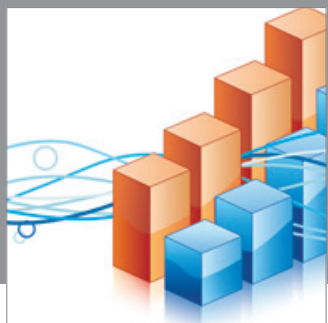

Advances in

Operations Research

mansans

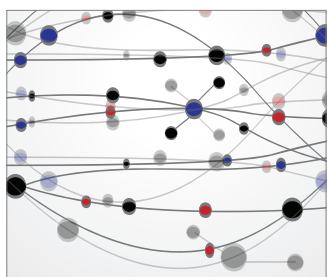

The Scientific World Journal
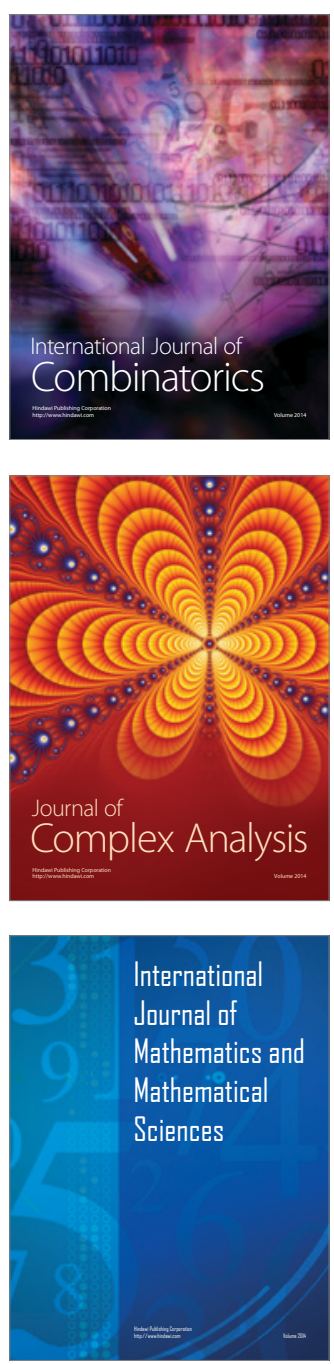
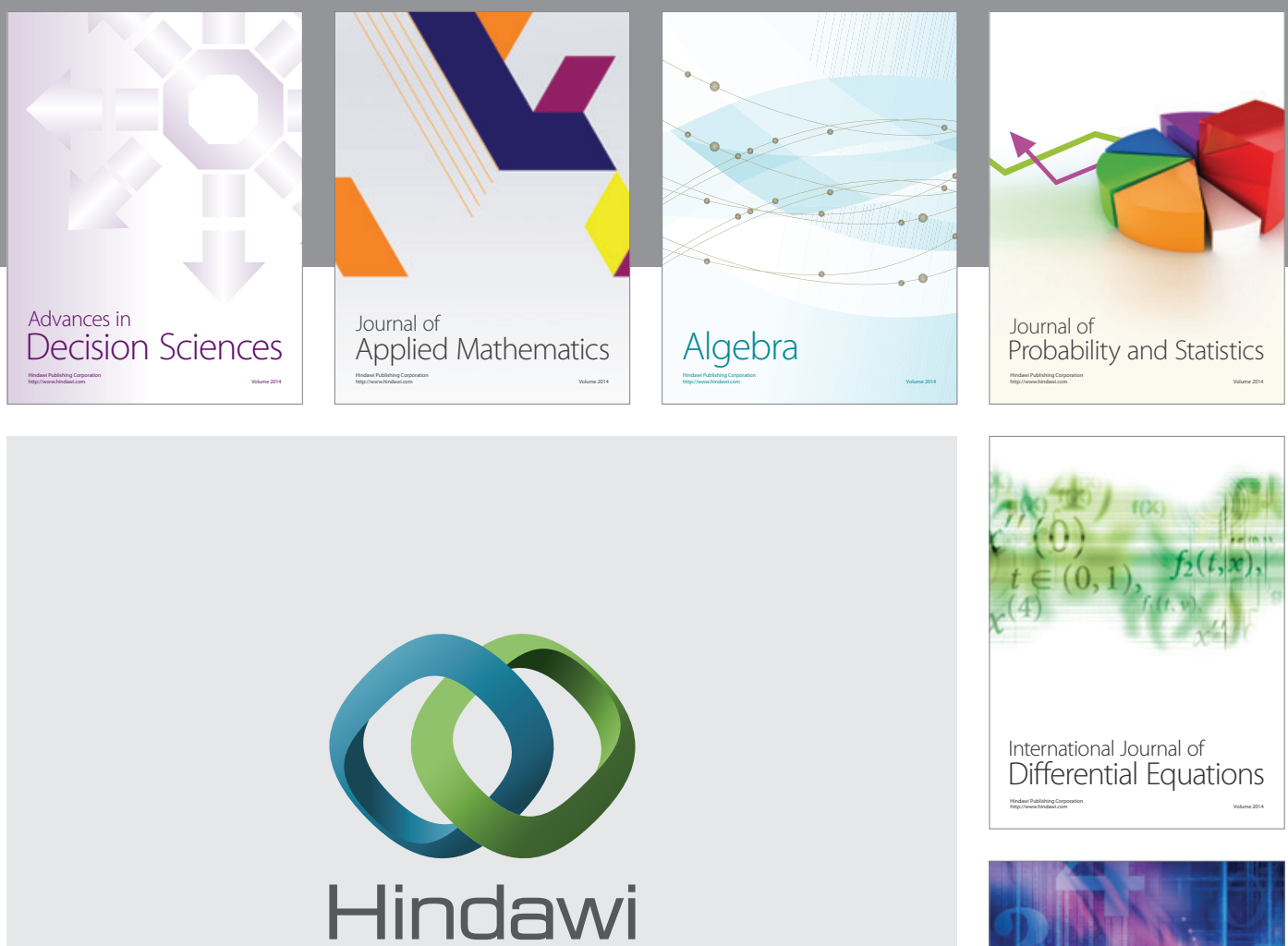

Submit your manuscripts at http://www.hindawi.com
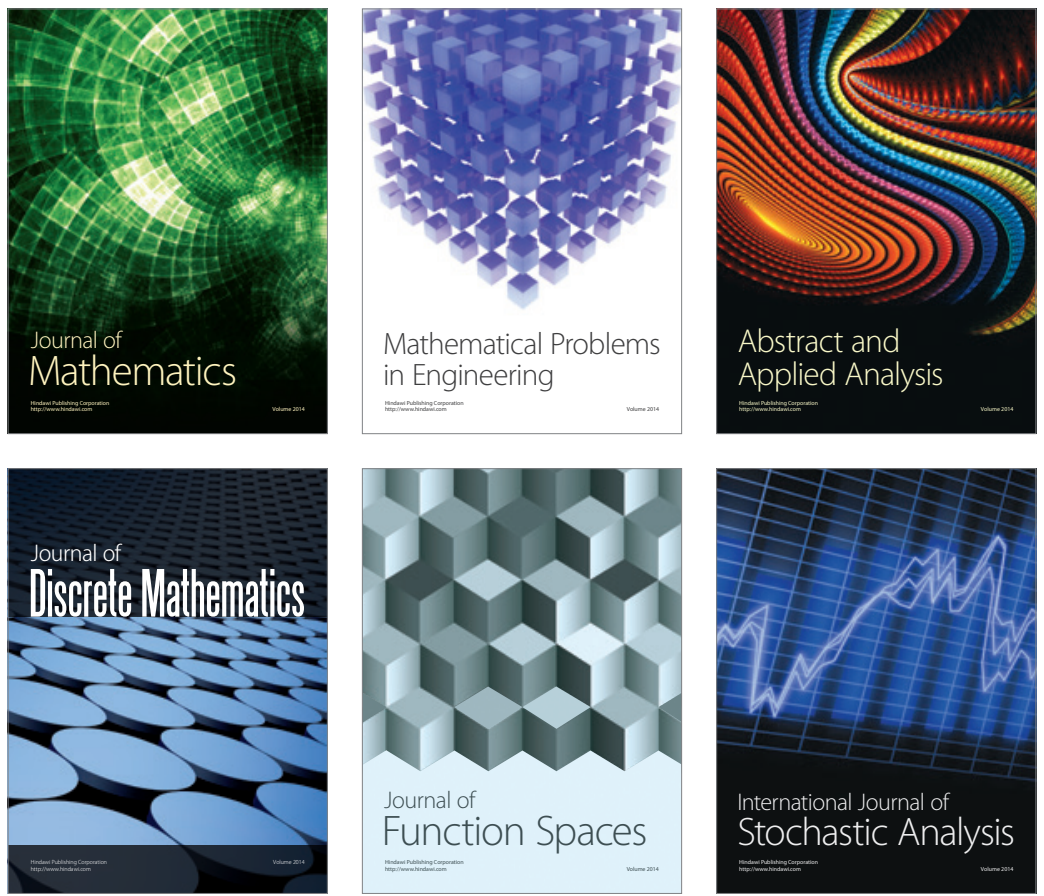

Journal of

Function Spaces

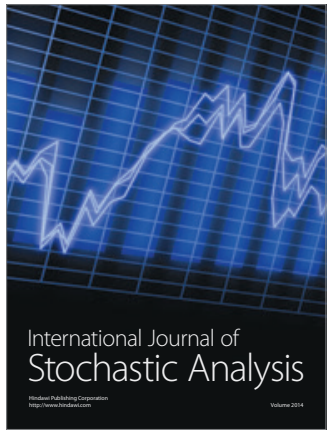

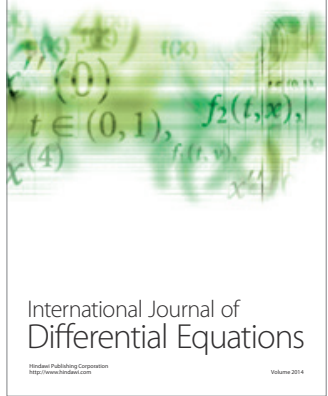
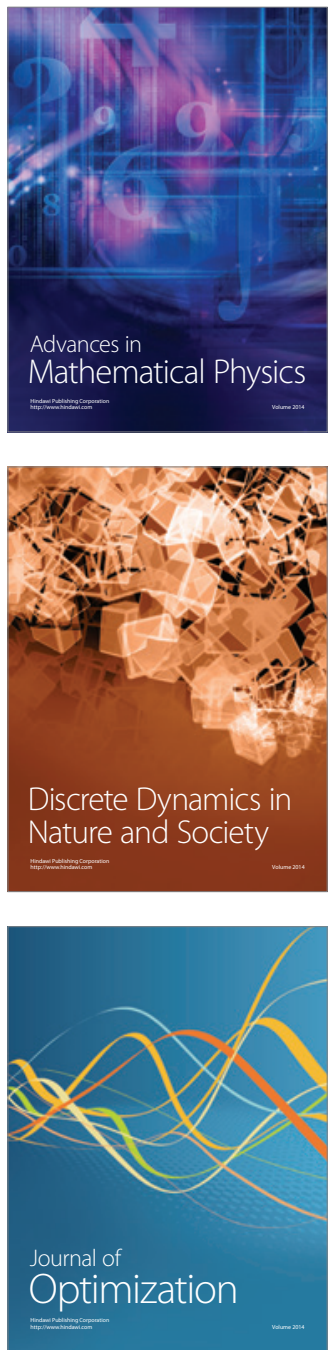